\title{
Modal Formulas True at Some Point in Every Model
}

\author{
Lloyd Humberstone \\ Monash University \\ lloyd.humberstone@arts.monash.edu.au \\ Received by Greg Restall \\ Published August 4, 2008 \\ http://www.philosophy .unimelb.edu.au/ajl/2008 \\ (C) 2008 Lloyd Humberstone
}

\begin{abstract}
:
In a paper on the logical work of the Jains, Graham Priest considers a consequence relation, semantically characterized, which has a natural analogue in modal logic. Here we give a syntactic/axiomatic description of the modal formulas which are consequences of the empty set by this relation, which is to say: those formulas which are, for every model, true at some point in that model.
\end{abstract}

\section{INTRODUCTORY OBSERVATIONS}

Graham Priest [Io] considers several alternative ways of formalizing the semantic ideas - in particular, Anekāntavāda ("non-one-sidedness") - in play in the Jain logic of what is sometimes called the classical period. One of these treatments involves models with items called facets to which truth is relativized In a spirit of egalitarian generosity, a formula is regarded as true tout court in a model if it is true relative to some facet in the model, and interest is focussed on the consequence relation defined by saying that $A$ (a formula) is a consequence of $\Gamma$ (a set of formulas) when any model in which all formulas in $\Gamma$ are true is a model in which $A$ is true. As is noted in [IO] the formal set-up is analogous to that of Kripke models for modal logic, but with facets playing the role of worlds $2^{2}$

\footnotetext{
${ }^{I}$ Another approach considered by Priest is a more traditional many-valued treatment, pursued earlier in Ganeri [3], one of the papers cited in [iO] and which can in turn be consulted for further historical references. (See also [9].) In more detail, it should be added that this manyvalued approach involves a 7-valued logic, while even Priest's modal-style treatment is "locally" - facet-by-facet, that is - 3-valued. As Priest has pointed out to me, some of the bivalent modal discussion that follows would be sensitive to the choice of designated elements if that aspect of his modal treatment had been adopted.

${ }^{2}$ Our models will be taken to be of the form $\langle W, R, V\rangle$ in which $W \neq \varnothing, R \subseteq W \times W$ and $V$ assigns to each propositional variable (sentence letter) a subset of $W$. Truth for an arbitrary for-
} 
The consequence relation just singled out, however, does not correspond to either of those usually attended to in modal logic: to the global consequence relation holding between $\Gamma$ and $A$ when every model - perhaps in some restricted class of models (for example, models whose accessibility relations are transitive) - in which the formulas in $\Gamma$ are true at every point (or every world) has A true at every world, or to the local consequence relation which would require in this instance that in any model (perhaps in a restricted class) any point at which all formulas in $\Gamma$ are true is a point at which $A$ is true. Priest's consequence relation, transposed into this modal setting, would instead have $A$ a consequence of $\Gamma$ just in case for any model (again perhaps subject to restriction), if for each formula in $\Gamma$ there is some point in the model at which that formula is true, then there is some point in the model at which $A$ is true. In view of the replacement of truth at all points by truth at at least one point, as property to be preserved (relative to any model), we might think of this novel consequence relation as the 'dual global' consequence relation.3

EXAMPLE I.I As an example, we note that - imposing no restriction whatever on the class of models $-\diamond \mathrm{q}$ has $\square \mathrm{p} \supset \mathrm{p}$ as a dual global consequence in the sense just described. To see this, we borrow the simple observation from Hughes [5] that in any model $\langle W, R, V\rangle$ no formula of the form $\square A \supset A$ can be false at both $x$ and $y$ when Rxy. (This observation is put to striking effect in the proof of Theorem II of [5]; themes from [5] have been subsequently taken up in [4] and [II.) Now suppose $\diamond q$ is true at some point $x$ in a model $\langle W, R, V\rangle$; this means that for some $y \in W, R x y$, so by the observation just recalled, $\square p \supset p$ must be true either at $x$ or at $y$ in the model, showing it to be a consequence in the current sense of $\diamond \mathrm{q}$.

While a study of the dual global consequence relation itself would certainly

mula $A$ at an element $x \in W$ is then given the usual inductive definition and will be written when convenient as " $\mathcal{M} \models_{x} A$ " in what follows, where $\mathcal{M}=\langle W, R, V\rangle$. As Allen Hazen has pointed out, the accessibility relations $R$ in such models do not correspond to anything in Priest's 'facets' models - though the picture in [IO could no doubt be enhanced to provide for an analogous ingredient. Thus what the models of $[\mathrm{IO}$ most directly suggest is models in which the accessibility relation is universal - determining the logic which in accordance with the notation in $\$ 3$ below would be called Sं5.

${ }^{3}$ Various other descriptions of what is going on at the level of valuations (truth-value assignments) come to mind. In the discussion after [I아, Greg Restall observed that truth in a model as the property to be preserved - is defined supervaluationally (resp. subvaluationally) in terms of truth at the model's points for the global (resp. dual global) consequence relation. In the terminology of $[7]$, the model-level valuation is obtained by taking the conjunctive (resp. disjunctive) combination of the point-level valuations in the two cases. 'Dual global consequence' is to be parsed as 'dual-global consequence' rather than 'dual global-consequence': that is, we are not here concerned with any of the candidates that might be considered for forming the dual of a consequence relation ([I2] and references therein - where the discussion is formulated in terms of consequence operations) - as applied to the global consequence relation. The threefold distinction globalllocalldual global here corresponds to the distinction globalllocallindefinite in [6., where, however, it was a classification of modal statements (or formulas) rather than consequence relations that was at issue. 
be of interest, in what follows we mainly concern ourselves - on the 'walk before you run' principle - only with the consequences of the empty set by this consequence relation. (Exception: the discussion surrounding Proposition I.3 below.) This is in keeping with the general tendency of work in modal logic to concentrate on logics construed very simple-mindedly as (certain) sets of formulas. We observe in passing that while the set of global and the set of local consequences of the empty set coincide, comprising simply the formulas true at every point in every model - for short, the valid formulas 4 - something new is obtained when we take instead the set of dual global consequences of the empty set: these are the formulas which are, for every model, true at some point of that model. Because of this last "some", we call such formulas $\exists$-valid. Variations on the Hughesian theme of Example I.I furnish us with several illustrations:

EXAMPLES I.2 $\quad$ (i) The formula $\diamond q \supset(\square p \supset p)$ is $\exists$-valid without being valid.

(ii) The formula $\square(\square p \supset p)$ is similarly $\exists$-valid but not valid.

(iii) Supposing we have available the truth and falsity constants $T$ and $\perp$, we can obtain an even shorter example than (ii), by substituting $\perp$ for $p$ and rewriting the result as: $\square \diamond \top$. (We could also have used $T$ in place of $q$ in (i) above, as well as in Example I.I.)

Some comments on the first and last of these examples are in order, beginning with the latter. Heuristically, it is useful to consider a standard example of a formula which can be true at a point in a model but which cannot be true at all points in any model: $\diamond \square \perp$. Since in any model this formula must be false at some point, we can take its negation as an example of a formula which must be true at some point in every model. This is the formula figuring in Example I.2(iii) 5

We turn to Example I.2(i). In view of Example I.I, the fact that this formula is $\exists$-valid raises the question whether the Deduction Theorem, in the sense of the $\Rightarrow$ direction of the 'Deduction Detachment Theorem' (or 'DDT'), as it is called in the abstract algebraic logic tradition:

(DDT)

$$
\Gamma, A \vdash B \Leftrightarrow \Gamma \vdash A \supset B,
$$

${ }^{4} \mathrm{~A}$ fuller discussion would require that we introduce the notion of validity on a frame, and discuss validity over this or that class of frames; similarly with the notion of $\exists$-validity introduced presently. To broaden the audience for this discussion, however, we keep restrict any mention of frames to the footnotes.

$5 \mathrm{~A}$ further observation which may be a mere curiosity is that the formulas appearing under I.2(i) and (ii) have both been associated with a property bearing the name 'quasi-reflexivity', here distinguished: $R$ is quasi-reflexive ${ }_{1}$ iff $\forall x, y(R x y \rightarrow R x x)$ and is quasi-reflexive $e_{2}$ iff $\forall x, y(R x y \rightarrow$ Ryy). I.2(i) and I.2(ii) then modally define the classes of frames whose accessibility relations are quasi-reflexive $_{1}$ and quasi-reflexive $e_{2}$, respectively. 
holds for $\vdash$ as the dual global consequence relation in modal logic. Call this relation $\Vdash_{D G}$. (Thus $A$ is $\exists$-valid just in case $\Vdash_{D G} A$, i.e., $\varnothing \Vdash_{D G} A$.) For the case of Examples I.I and I.2(i), $\Gamma=\varnothing$, and $A$ and $B$ are respectively $\nabla q$ and $\square p \supset p$. It is not hard to see that the Deduction Theorem is indeed satisfied in full generality by $\vdash_{\mathrm{DG}}$ :

PROPOSIT ION I.3 For any set $\Gamma$ of formulas and any formulas A, B:

$$
\Gamma, \mathrm{A} \Vdash_{\mathrm{DG}} \mathrm{B} \text { implies } \Gamma \Vdash_{\mathrm{DG}} \mathrm{A} \supset \mathrm{B} \text {. }
$$

Proof: Suppose $\Gamma, A \Vdash_{\mathrm{DG}} \mathrm{B}$, and we have a model $\mathcal{M}=\langle\mathrm{W}, \mathrm{R}, \mathrm{V}\rangle$ each formula in $\Gamma$ being true in $\mathcal{M}$ at some point or other (not necessarily the same point for different formulas). We must show that there is some element of $W$ at which $A \supset B$ is true. Pick any $x \in W$. If $\mathcal{M} \models_{x} A \supset B$ (notation as in note 2), then we are done. So suppose $\mathcal{M} \forall_{x} A \supset B$. Then $\mathcal{M} \models_{x} A$ (and $\mathcal{M} \forall_{x} B$, though we don't need this). Since we already knew that each formula in $\Gamma$ is true at some point or other in $\mathcal{M}$, we can now extend this same claim to the case of $\Gamma \cup\{A\}$. As $\Gamma$, $A \Vdash_{D G} B$, there is accordingly some $y \in W$ for which $\mathcal{M} \models_{y} B$. Thus $\mathcal{M} \models_{y} A \supset B$, and we are done.

Unusually, the fate of (DDT) for $\Vdash_{\mathrm{DG}}$ is settled negatively not, as we have just seen, by a failure of its $\Rightarrow$ ('Deduction') half, but by its $\Leftarrow$ ('Detachment') half. For taking $\Gamma$ as $\{A \supset B\}$, securing the right-hand side of (DDT), its $\Leftarrow$ direction would give $A, A \supset B \Vdash_{D G} B$, which in turn implies, by the 'Cut', or transitivity, property of consequence relations the (considerably weaker) conclusion that $\Vdash_{\mathrm{DG}} A$ and $\Vdash_{\mathrm{DG}} A \supset \mathrm{B}$ together imply that $\Vdash_{\mathrm{DG}} \mathrm{B}-\mathrm{a}$ conclusion whose falsity will be shown in Example 2.I below.

Returning to from the vagaries of the consequence relation $\Vdash_{\mathrm{DG}}$ in its full glory to the specific topic $\exists$-valid formulas, we conclude with another example of such a formula which again fails to be valid, as in Examples I.2, but which is of a lower modal degree than all the cases listed there.

EXAMPLE I. 4 The formula $p \supset \square$ is $\exists$-valid but not valid. The falsity of this conditional at a point implies the falsity of its consequent and hence the falsity of $p$ at some accessible point, at which accordingly the conditional itself is true. So the conditional cannot be false throughout a model.

Some of these examples we have seen here will receive further attention in the following section (in the discussion following Proposition 2.3).

\section{AXIOMATIZING THE $\exists$-VALID FORMULAS}

We begin by recalling some established terminology for discussing modal logics as sets of formulas. First, we are working in a language with a countable supply of propositional variables (or sentence letters), amongst which three are $p, q$, and $r$, and any functionally complete supply of boolean connectives (the notation here is $\neg, \wedge, \vee, \supset$, $\equiv$, with the usual arities and interpretations) along 
with the I-ary modal connective $\square$ (regarding $\diamond$ as abbreviating $\neg \square \neg$ ). A modal logic is set of formulas of this language which contains all truth-functional tautologies and is closed under uniform substitution (of arbitrary formulas for propositional variables) and Modus Ponens (equivalently in this setting: under truth-functional consequence). A modal logic is normal if also contains the formula $\square(p \supset q) \supset(\square p \supset \square q)$ and is closed under the rule (of Necessitation): From $A$ to $\square A$. The smallest normal modal logic is called $K$, the above description amounting to one particular axiomatization of it. A modal logic $\mathrm{S}$ is quasi-normal if $\mathrm{K} \subseteq \mathrm{S}$; such a logic may fail to be normal because it may not itself be closed under Necessitation. We need also the following non-standard terminology. A modal quasi-logic is defined exactly as per the definition of 'modal logic' above but without the requirement of closure under Modus Ponens (equivalently, as we observed, the requirement of closure under truth-functional consequence). When $S$ is any modal quasi-logic (which includes the case of $\mathrm{S}$ a modal logic) which has been characterized axiomatically - i.e., as the least quasi-logic containing given formulas (axioms) and closed under given rules - we write $\vdash_{S} A$ for $A \in S$ and refer to $A$ as a theorem, or provable formula, of $\mathrm{S}$.

Since every valid formula is $\exists$-valid, letting " $\mathrm{K}$ " be a name for our as yet unaxiomatized system whose theorems are to coincide with the $\exists$-valid formulas, we will have $\dot{K} \supseteq \mathrm{K}^{6}$ By Examples I.2, we have, more specifically, $\dot{\mathrm{K}} \supsetneq \mathrm{K}$. It is not hard to see that the set of $\exists$-valid formulas is not closed under $\mathrm{Ne}$ cessitation - for example, prefixing a $\square$ to the formula in Example I.4 yields a formula which is not $\exists$-valid.7 One might jump to the conclusion that $\mathrm{K}$ is a proper quasi-normal modal logic, i.e., a modal logic which is quasi-normal but not normal. But this would be premature, because the existential quantification in the definition of $\exists$-validity has the effect that we are not dealing with a modal logic at all, but with a ('proper') modal quasi-logic.

EXAMPLE 2.I $A_{1}=\square(\square p \supset p)$, from Example I.2(ii) and a relettered version $A_{2}=\square(\square q \supset q)$ are both $\exists$-valid, while $A_{1} \wedge A_{2}$ is not. So the set of $\exists$-valid formulas is not closed under truth-functional consequence. To reformulate this as a counterexample to closure under Modus Ponens, we have $A_{1} \supset\left(A_{2} \supset\left(A_{1} \wedge A_{2}\right)\right) \exists$-valid, so two applications of Modus Ponens, each of the antecedents here being $\exists$-valid, take us out of the class of $\exists$-valid formulas, from which it follows that the class of such formulas is not closed under Modus Ponens. (In fact, the result of the first envisaged application, namely $A_{2} \supset\left(A_{1} \wedge A_{2}\right)$ is $\exists$-valid, so it is the second application, specifically, that illustrates the non-closure claim. The $\exists$-validity of $A_{2} \supset\left(A_{1} \wedge A_{2}\right)$ is indicative of another point, namely that whenever $\mathrm{D}$ is a truth-functional consequence

\footnotetext{
${ }^{6} \mathrm{We}$ recall from note 2 that, as is standard, the ' $W$ ' of our models is always non-empty.

${ }^{7}$ Consider any model $\langle W, R, V\rangle$ with $|W| \geqslant 2, R=W \times W$ and $\varnothing \subsetneq V(p) \subsetneq W . \diamond(p \wedge \diamond \neg p)$ is true throughout the model; but this formula is equivalent to the negation of the formula, $\square(p \supset \square p)$, currently under consideration.
} 
of (any single formula) C - when C $\supset$ D is a substitution instance of a tautology, that is - if $C$ is $\exists$-valid, then so is $D$. This is an immediate consequence of the definition of $\exists$-validity, as indeed is the more general formulation that whenever $\vdash_{K} C \supset D$, if $C$ is $\exists$-valid, then $D$ is too. In the present case, $C$ is $A_{1}$ and $D$ is $A_{2} \supset\left(A_{1} \wedge A_{2}\right)$.)

Not only do reletterings, as in the above example, preserve $\exists$-validity, but arbitrary (uniform) substitutions do so, as is easily seen. (In outline: if $A(B)$, arising from the substitution of $B$ for $p$ (say), is not $\exists$-valid there is a model $\langle W, R, V\rangle$ at every point in which $A(B)$ is false. Make a new model by changing (only) $V$ to $V^{\prime}$ with $V^{\prime}(p)$ being the set of points at which $B$ is true in $\langle W, R, V\rangle$.) Thus extending the definition of quasi-normality so that it can apply to quasilogics (whose theorems include all those of $\mathrm{K}$ ), our desired $\dot{K}$ would qualify as a 'quasi-normal modal quasi-logic'. One way to axiomatize a quasi-normal modal logic is to take as axioms all the theorems of some normal modal logic, along with new axioms, and use Modus Ponens and uniform substitution - but not Necessitation - as the rules. The smallest modal logic extending $\mathrm{K}$ by the axiom $\mathrm{T}=\square \mathrm{p} \supset \mathrm{p}$ is axiomatized in this way in Segerberg [II] $]^{8}$ with $\mathrm{T}$ in the role of 'new axiom'. Its theorems are the formulas true at every reflexive point in every model (by contrast with KT, the least normal modal logic containing T, whose theorems are the formulas true at every point in every reflexive model). 9 The corresponding strategy here will have to be even more restrictive, since as we have seen, Modus Ponens can destroy $\exists$-validity. Accordingly, we replace Modus Ponens (in the axiomatization of $\dot{K}$ ) with another rule, or family of rules, namely those given here as $(\dot{\mathrm{K}})_{n}$ for each $n \in N a t$, with $\diamond^{i}$ indicating a string of $i$ occurrences of $\diamond$ :

$(\dot{K})_{n} \quad \frac{A \vee \diamond A \vee \diamond^{2} A \vee \ldots \vee \nabla^{n-1} A}{A}$

The labelling has been chosen so that $(\dot{K})_{n}$ is the rule having a disjunctive pre-

\footnotetext{
${ }^{8}$ See pp. I7 $8 f$. of [II]. A typo at line 2 of p. 178 has resulted in the appearance of an unwanted " $\diamond$ ". With this correction, $T$ appears (in schematic form) as " $T^{n}$ " for $n=0$ there. We should remark that in fact the rule of uniform substitution is not part of Segerberg's axiomatizations, since he uses schemata rather than individual axioms and takes $T$ as the schema $\square A \supset A$. We will similarly avoid any mention of this rule in the axiomatization of $\dot{K}$ below, on the basis of which axiomatization the rule is nevertheless admissible.

${ }^{9} \mathrm{~A}$ reflexive point is a point accessible to itself and a reflexive model is one in which all points are reflexive. In a notation like that of Segerberg [II], disregarding the superscript - see the previous note - this would be called $\mathrm{K}[\mathrm{T}]$. The semantic characterization of this logic given in $[\mathrm{II}]$ is different from that just given (though equivalent), being put there in terms of models with distinguished elements - truth at which is what matters for validity and all of which have to be reflexive for the class of models w.r.t. which $\mathrm{K}[\mathrm{T}]$ is sound and complete. (A referee has complained of the 'deplorable prolixity' involved in referring to KT by that name rather than simply as T. I prefer not to use labels which are ambiguous as between referring to modal principles and to modal logics, however, and accordingly follow the nomenclature of [2] in this respect.)
} 
miss with $n$ disjuncts. ${ }^{\circ}$ Sometimes in what follows we write " $\nabla^{0} A$ " in place of A for the first disjunct.

LEMMA 2.2 For each $\mathrm{n}$, the rule $(\dot{\mathrm{K}})_{\mathrm{n}}$ preserves $\exists$-validity.

Proof: Suppose the premiss for an application of $(\dot{K})_{n}$ is $\exists$-valid. Thus any model contains a point verifying at least one of the disjuncts, say $\nabla^{i} A$, of this premiss, in which case in $i$ steps of the relation $R$ from that point is a point at which $A$ is true $(0 \leqslant i \leqslant n)$. Thus $A$, the conclusion of this application of $(\dot{K})_{n}$, is $\exists$-valid.

As in the case of the usual proof that Necessitation preserves validity, the above proof shows more than what the lemma claims, since it shows that any model in which a premiss for the rule is true somewhere, is itself a model (not just there is some model or other) in which the conclusion is true somewhere.

We now have all we need to present our axiomatization of K:

Axioms: All theorems of K. $\quad$ RULEs: $(\dot{K})_{n}$ for each $n \in$ Nat.

This axiomatization may seem artificial, unenlightening and cumbersome, e.g., in respect of the number of axioms and rules. It is convenient to work with for the moment, however, and we postpone looking at simpler alternatives until Section 3 (especially Proposition 3.2). Some words are in order on what might seem to be an alternative - though not simpler, by any customary criterion - to those with expectations grounded in modal logics as opposed to modal quasilogics. The expectation might be that we can replace the rules $(\dot{K})_{n}$ with rules having instead several schematic letters in the premiss-disjuncts, disjoined in the conclusion, as we illustrate here for a variant along these lines of $(\dot{\mathrm{K}})_{3}$ :

$$
\frac{A \vee \diamond B \vee \diamond \diamond C}{A \vee B \vee C}
$$

One might think that using this evidently $\exists$-validity-preserving rule in place of $(\dot{K})_{3}$ does not result in the loss of any $\dot{K}$ theorems, since we can recover the effect of $(\dot{K})_{3}$ by taking the special case in which $C=B=A$, giving conclusion $A \vee A \vee A$ which we simplify to obtain the original $(\dot{K})_{3}$ conclusion $A$. In fact, though, there is no machinery available to effect the simplification just alluded to in the current quasi-logical setting. The envisaged variant of our axiomatization (with rules like that inset above replacing all the $\left.(\dot{K})_{n}\right)$ delivers as theorems at most the $\mathrm{K}$-theorems and those disjunctive formulas which are $\exists$-valid but not valid.

Another alternative to the rules $(\dot{K})_{n}$ would allow greater freedom on the iterations of $\diamond$ in the premiss, not insisting instead that they be o, I, 2, etc., but allowing that they be any $k_{1}, \ldots, k_{m} \in N a t$.

\footnotetext{
${ }^{\text {Io }}$ Of course literally speaking like the premiss is either just $A$ or is a binary disjunction selected as desired, so here what is meant by "disjuncts" is the formulas $A, \diamond A, \diamond^{2} A$, appearing as disjuncts in the above unbracketed representation of a $(\dot{K})_{n}$ premiss.
} 
Liberalized $(\dot{\mathrm{K}})$ rules:

$$
\frac{\diamond^{k_{1}} A \vee \diamond^{k_{2}} A \vee \ldots \vee \diamond^{k_{m}} A}{A}
$$

These rules, one for each $\left\langle k_{1}, \ldots, k_{m}\right\rangle$, are again $\exists$-validity preserving, and have the original rules $(\dot{K})_{n}$ as special cases. They could therefore be used in the axiomatization of $\dot{K}$ without affecting the class of theorems - though they are not in general derivable, but only admissible, in the axiomatization given above. That axiomatization turns out to be the most convenient for the completeness proof below (Proposition 2.5). But let us begin with the issue of soundness.

PROPOSITION 2.3 (Soundness.) Every theorem of $\dot{\mathrm{K}} i s \exists$-valid.

Proof: Since all K-theorems are $\exists$-valid, the result follows by Lemma 2.2.

Of course we should also like to establish that the converse of Proposition 2.3 holds. To inspire confidence, as well as to make some observations of interest in their own right, we revisit some earlier examples of $\exists$-valid formulas. Let us look at Examples I.2(ii) and I.4.

For the first of these the formula concerned is $\square(\square p \supset p)$. An easy semantic check reveals that the following disjunction is valid and hence K-provable:

$$
(\square \mathrm{p} \supset \mathrm{q}) \vee \square(\square \mathrm{r} \supset \mathrm{p}) \text {. }
$$

(It may be helpful to think of the first disjunct as following from $\neg \square \mathrm{p}$ and the second from $\square$ p.) A well-known admissible rule of $K$ (derivable on the basis of the axiomatization mentioned above) takes us from any provable disjunction $A \vee B$ to $\square A \vee \diamond B$. Applying this rule to our disjunction gives:

$$
\square(\square \mathrm{p} \supset \mathrm{q}) \vee \diamond \square(\square \mathrm{r} \supset \mathrm{p}) \text {, }
$$

which is therefore K-provable, along with all its substitution instances, and in particular the result of substituting $p$ for $q$ and $r$. But this last formula now has the form of a premiss for $(\dot{\mathrm{K}})_{2}$.

In the case of Example I.4, the formula concerned is $p \supset \square$. Again we note the K-provability of the 'variegated' disjunction

$$
(\mathrm{q} \supset \square \mathrm{p}) \vee \diamond(\mathrm{p} \supset \square \mathrm{r}),
$$

and again we replace the extraneous variables $q$ and $r$ by $p$ to obtain a premiss to apply $(\dot{\mathrm{K}})_{2}$ to. The conclusion of that application is the desired formula.

To put these examples into a more general light let us, inspired by Example I.I, call A a Hughes formula if for any point in any model, $A$ is true at that point or at all points accessible to that point. And similarly let us call $A$ a weak Hughes formula if for any point in any model $A$ is true at that point or else at some point accessible to it. In other words, $A$ is a Hughes formula (weak Hughes formula) just in case $A \vee \square A$ (resp. $A \vee \diamond A$ ) is valid. Note that Hughes formulas are not in general $\exists$-valid, while weak Hughes formulas are (by $(\dot{K})_{2}$, in view of Lemma 2.2 and the fact that validity implies $\exists$-validity). Example I.4 (p $\supset \square$ ) is a weak 
Hughes formula, accounting for its $\exists$-validity. (Of course a formula which is $\exists$-valid need not be a weak Hughes formula; example: $p \supset \square \square p$ - if false at a point this must be true at some successor of a successor, rather than at some successor the falsifying point itself.) As [5], recalled in Example I.I, observed, $\square p \supset p$ is a Hughes formula. While, as just noted, not all such formulas are $\exists$ valid, it is not hard to see that whenever $A$ is a Hughes formula, $\square A$ and (for any formula $B) \diamond B \supset A$ are weak Hughes formulas and hence $\exists$-valid, accounting for Examples I.2(i) and (ii).

We return to the project of showing that $\dot{K}$, as axiomatized above, is adequate to its appointed task of yielding all the $\exists$-valid formulas. Strictly speaking, it is the "if" half of the following result that serves as a lemma for the completeness theorem ${ }^{m}$ (though the discussion in the following section draws on the characterization of Kं-provability provided by the full result).

LEMMA 2.4 For any formula $A, \vdash_{\dot{\mathrm{K}}} A$ if and only if for some $\mathrm{n} \in \mathrm{Nat}, \vdash_{\mathrm{K}} \diamond^{0} \mathrm{~A} \vee \ldots \vee$ $\diamond^{n} A$.

Proof: The "if" direction follows from the fact that if the cited disjunction is $\mathrm{K}$-provable, it is an axiom of $\dot{\mathrm{K}}$, so one application of the rule $(\dot{\mathrm{K}})_{\mathrm{n}}$ gives the result.

For the "only if" direction, suppose that $\vdash_{\dot{K}} A$. We show that there is a proof of $A$ from the above axiomatization in which the only one of the rules $(\dot{K})_{n}$ is applied and in which it is applied only once. Suppose otherwise, so that a proof involves an application of $(\dot{\mathrm{K}})_{m}$ to a $\dot{K}$-axiom (i.e., K-theorem) followed by a further application of $(\dot{K})_{n}$, where possibly $m=n$ (and possibly not). Let $B$ be the intermediate conclusion. So $B$ was obtained from $\diamond^{0} B \vee \ldots \vee \diamond^{m} B$, and $B$ itself is of the form $\diamond^{0} A \vee \ldots \vee \diamond^{n} A$. Thus the axiom in question had the form

$$
\bigvee_{i=0}^{n} \diamond^{i}\left(\bigvee_{j=0}^{m} \nabla^{j} A\right)
$$

and distributing the $\diamond \mathrm{s}$ across the $\vee \mathrm{s}$ in $\mathrm{K}$, we could equally well have begun with the following K-theorem

$$
\bigvee_{i=0}^{n} \bigvee_{j=0}^{m} \diamond^{i} \diamond^{j} A
$$

or in other words:

$$
\bigvee_{i=0}^{n} \bigvee_{j=0}^{m} \diamond^{i+j} A
$$

Eliminating any repeated disjuncts and re-ordering the disjuncts, this means that we have as K-provable:

\footnotetext{
${ }^{\text {II }}$ This was pointed out to me by Allen Hazen, who observes that stating the lemma only in that direction would enable one to obtain the "only if" direction as a corollary to Theorem 2.5, if desired.
} 


$$
\diamond^{0} A \vee \ldots \vee \diamond^{m+n} A
$$

from which we obtain the desired conclusion, $A$, by one application of $(\dot{\mathrm{K}})_{\mathrm{m}+\mathrm{n}+1}$.

PROPOSITION 2.5 (Completeness.) Every $\exists$-valid formula is a theorem of $\dot{\mathrm{K}}$.

Proof: Suppose that $\nvdash_{\dot{K}} A$. We must find a model at every point of which $A$ is false, showing $A$ not to be $\exists$-valid. As $\nvdash_{\dot{K}} A$, the following set of formulas is K-consistent: $\left\{\neg A, \square \neg A, \square \square \neg A, \ldots, \square^{k} \neg A, \ldots\right\}$. Otherwise some finite subset, which without loss of generality we may take to be $\left\{\neg A, \square \neg A, \ldots, \square^{n} \neg A\right\}$, for some $n$, would be K-inconsistent, which implies that $\vdash_{K} \diamond^{0} A \vee \ldots \vee \diamond^{n} A$, contradicting the supposition that $\nvdash_{\dot{\mathrm{K}}} A$, by Lemma 2.4. Thus in, e.g., the canonical model for $K$ there is a point at which each of $\neg A, \square \neg A, \square \square \neg A, \ldots$, $\square^{\mathrm{k}} \neg A, \ldots$ is true. Taking the submodel generated by that point we get a model at every point in which $A$ is false.

Thus, combining Propositions 2.3 and 2.5, we have the desired conclusion that $\exists$-validity and $\dot{K}$-provability coincide.

\section{VARIATIONS AND EXTENSIONS}

Instead of looking at formulas true at some point in every model, we could for example consider those formulas true at some point in every model with a reflexive accessibility relation. We could axiomatize a modal quasi-logic KंT as we axiomatized $\dot{K}$ in the preceding section, except for allowing as axioms all theorems of the normal modal logic KT, rather than just those of K. We would of course lose some of our examples of formulas which are $\exists$-valid though not valid (reconstruing these notions with a reflexivity condition in force), including all of Examples I.2 and the conjunctive formula in Example 2.I. A completeness result in this case could be obtained exactly along the lines of Proposition 2.5 , mutatis mutandis. And an obvious simplification of the rules $(\dot{K})_{n}$ would be available since $\vdash_{K T}\left(A \vee \diamond A \vee \ldots \diamond^{n-1} A\right) \equiv \diamond^{n-1} A$, so we could drop all but the last disjunct of their premisses. Similarly, in $\dot{S} 4$ ( = K KT4, where 4 is $\square p \supset \square \square p$ ), all these simplified rules could be collapsed to one, with premiss schematically represented as $\diamond A$, and conclusion $A$. (Presently we shall see - Proposition 3.2 - that the rules $(\dot{K})_{n}$ can be replaced by a single rule even for $\dot{K}$ itself.)

What if we wanted, however, to provide a modal quasi-logic extending by the rule $(\dot{\mathrm{K}})_{n}$ a normal modal logic which was not decidable? A treatment in the style here contemplated for KंT would not deserve the name of an axiomatization, since - a standard observation, here - without a recursive set of axioms, mechanical proof checking is not possible. In that case, one can instead employ any axiomatization of the original logic together with the rules $(\dot{\mathrm{K}})_{n}$ subject to 
a 'global derivational constraint ${ }^{12}$ forbidding the application of the one of the latter rules before any application of a rule which is not one of them. A similar axiomatization could be given for $\dot{K}$ itself, if the basis given in Section 2 is felt to be unwieldy or uninformative with its vast suite of axioms.

We can also reduce the number of distinctive rules used in that basis, since the collection of rules $\left\{(\dot{\mathrm{K}})_{\mathfrak{n}} \mid \mathrm{n} \in \mathrm{Nat}\right\}$ can be replaced by the single rule $(\dot{\mathrm{K}})_{2}$ which figured in our working through of earlier examples in the discussion after Proposition 2.3 (though this rule will typically need to be applied more than once, by contrast with the earlier $\left\{(\dot{K})_{n}\right)$. By contrast with the axiomatization of $\dot{K}$ with the full panoply of $(\dot{K})_{n}$ rules, this one will typically have to be used several times in a proof ${ }^{13}$ We illustrate this with the case of $(\dot{\mathrm{K}})_{4}$, with premiss

$$
A \vee \diamond A \vee \diamond \diamond A \vee \diamond \diamond \diamond A
$$

Instead of obtaining $A$ by applying $(\dot{\mathrm{K}})_{4}$, we reformulate the premiss as the K-equivalent:

$$
((A \vee \diamond A) \vee \diamond(A \vee \diamond A)) \vee \diamond(((A \vee \diamond A) \vee \diamond(A \vee \diamond A)))
$$

and apply $(\dot{K})_{2}$, three times to derive $A$. We must check that this strategy is always available.

Consider two sequences of formulas based on a given formula $A$. First let $P_{n}(A)$ be a premiss for $(\dot{K})_{n}$, i.e., be the formula $A \vee \diamond^{0} A \vee \ldots \vee \diamond^{n-1} A$. Secondly define $Q_{n}(A)$ thus:

$$
\mathrm{Q}_{0}(A)=A ; \quad \mathrm{Q}_{n+1}(A)=\mathrm{Q}_{\mathfrak{n}}(A) \vee \diamond \mathrm{Q}_{\mathfrak{n}}(A) .
$$

By induction on $n$, we have:

LEMMA 3.I For any formula $A$, and any $n \in N a t, \vdash_{K} P_{n}(A) \equiv Q_{n}(A)$.

PROPOSITION 3.2 If all the rules $(\dot{\mathrm{K}})_{n}$ except for $(\dot{\mathrm{K}})_{2}$ are dropped from the axiomatization of $\dot{\mathrm{K}}$ given in Section 2, all theorems of $\dot{\mathrm{K}}$ remain provable.

Proof: If $A$ is an axiom in the original basis, it is an axiom in the reduced basis currently envisaged. We know from the proof of Lemma 2.4 that if one of the $(\dot{K})_{n}$ rules has been applied, there is a proof of $A$ in which there is only one such application. If $n=1, A$ is an axiom and no such application is required. If $n=2$, we apply $(\dot{K})_{2}$ from the reduced basis. If $n>2$ we make repeated applications of $(\dot{K})_{2}$ as follows. Replace the K-provable premiss $P_{\mathfrak{n}}(A)$ of a single application of $(\dot{K})_{n}$ by $Q_{n}(A)$, also K-provable by Lemma 3.I. Apply $(\dot{K})_{2}$ $n-1$ times to get a proof of $A$ from the new basis.

\footnotetext{
${ }^{12}$ The phrase is George Lakoff's, from the late r96os, and has nothing to do with the semantic local/global contrast mentioned in our opening section. See $[8]$, p. 72 , for further references and applications to logic.

${ }^{13}$ Recall that the proof of Lemma 2.4 showed at most one appeal needed to be made to these rules in any proof - which we can formulate as the claim that every provable formula has a proof in which there is exactly one such appeal, since we can use $(\dot{K})_{1}$ in the absence of another $(\dot{\mathrm{K}})_{\mathrm{n}}$-application.
} 
Another application of Lemma 3.I is to the question of which normal modal logics are themselves already closed under all the rules $(\dot{K})_{n}(n \in N a t)$, since from it we infer that this is equivalent to being closed under the single rule $(\dot{K})_{2}$. The question is then easily answered since the latter is in turn equivalent (for any modal logic, in fact) to having as a theorem $p \supset \square$. Not that this comes as a surprise, since the smallest normal modal logic (and we could equally well say 'quasi-normal' here) containing this formula, $\mathrm{KT}_{\mathrm{c}}$ in the notation of Chellas $[2]$, is sound and complete w.r.t. the class of one-point models, restricted to which the concepts of $\exists$-validity and validity coincide.

I would like to close with the following observations from Allen Hazen. Hazen observes that one could define the $\exists$-validity of a first order (non-modal) open formula to be equivalent to the validity of its existential closure is. The set of $\exists$-valid formulas is then analogous to the set of modal formulas true at some point in every model (the theorems of $\dot{S} 5$, mentioned in note 2 ), and from Herbrand's Theorem one can obtain the following characterization of these formulas. A formula is $\exists$-valid if and only if some disjunction of alphabetic variants of it and its partial $\exists$-closures is valid, where by a partial $\exists$-closure of a first order formula is meant the result of binding some or all of its free variables by initial existential quantifiers. As Hazen remarks, this characterization is suggestive of our rules $(\dot{K})_{n}$ (though as I would add: these rules admit of the simplification mentioned above à propos of $\dot{S} 4$, to the single rule $\nabla A / A) .{ }^{14} \mathrm{He}$ further draws attention to predicate-logical cases analogous to those under Example 2.I which show these first order $\exists$-valid formulas are not closed under conjunction or Modus Ponens. The open formula $(F x \rightarrow \forall x$. Fx $) \wedge(G x \rightarrow \forall x . G x)$, for instance, is not $\exists$-valid, though each of its conjuncts is.

\section{ACKNOWLEDGMENTS}

I am grateful to Graham Priest for making available the written version of [10], and to him and Allen Hazen for all their comments, as well as to two $A \mathcal{F L}$ referees for helpful corrections.

\section{REFERENCES}

[I] P. Balbiani, I. Shapirovsky, and V. Shehtman, 'Every World Can See a Sahlqvist World', pp. $69-85$ in G. Governatori, I. Hodkinson and Y. Venema (eds.), Advances in Modal Logic, Volume 6, College Publications, London 2006.

[2] B. F. Chellas, Modal Logic: An Introduction, Cambridge University Press, Cambridge i980.

${ }^{14}$ This corresponds to the fact that re-construed as first order formulas, modal formulas have exactly one free variable. 
[3] J. Ganeri, 'Jaina Logic and Philosophical Problems', History and Philosophy of Logic, Vol. 23 (2003), 267-28I.

[4] R. Goldblatt, I. Hodkinson, and Y. Venema, 'Erdōs Graphs Resolve Fine's Canonicity Problem', Bulletin of Symbolic Logic, Vol. IO (2004), I86208.

[5] G. E. Hughes, 'Every World Can See a Reflexive World', Studia Logica, Vol. 49 (I990), I74-I8I.

[6] Lloyd Humberstone, 'Necessary Conclusions', Philosophical Studies, Vol. 4I (I98I), 32I-325.

[7] Lloyd Humberstone, 'Classes of Valuations Closed Under Operations Galois-Dual to Boolean Sentence Connectives', Publications of the Research Institute for Mathematical Sciences, Kyoto University, Vol. 32 (1996), 984. (Available at the time of this writing from http://projecteuclid. org/euclid.prims/1195163180)

[8] Lloyd Humberstone, 'Extensions of Intuitionistic Logic Without the Deduction Theorem: Some Simple Examples', Reports on Mathematical Logic, Vol. 40 (2006), 45-82. (Available at the time of this writing from http://www.iphils.uj.edu.pl/rml/rml-4o/o3-humberstone.pdf)

[9] Pragati Jain, 'Saptabhaing - The Jaina Theory of Sevenfold Predication: A Logical Analysis', Philosophy East and West, Vol. 50 (2000), 385-399.

[Io] Graham Priest, 'Jaina Logic - a Contemporary Perspective', unpublished paper (given as a talk at the Melbourne University Logic Seminar in September, 2006).

[II] Krister Segerberg, An Essay in Classical Modal Logic, Filosofiska Studier, Uppsala I97I.

[12] M. Spasowski, 'Some Connections Between $C n$ and $C n^{-1}$ and $d C n$ ', Bulletin of the Section of Logic, Vol. 2 (1973), 46-50. 
The Australasian fournal of Logic (ISSN I448-5052) disseminates articles that significantly advance the study of logic, in its mathematical, philosophical or computational guises. The scope of the journal includes all areas of logic, both pure and applied to topics in philosophy, mathematics, computation, linguistics and the other sciences.

Articles appearing in the journal have been carefully and critically refereed under the responsibility of members of the Editorial Board. Only papers judged to be both significant and excellent are accepted for publication.

The journal is freely available at the journal website at

$$
\text { http://www.philosophy.unimelb.edu.au/ajl/ }
$$

All issues of the journal are archived electronically at the journal website.

SuBSCRIPTIONS Individuals may subscribe to the journal by sending an email, including a full name, an institutional affiliation and an email address to the managing editor at ajl-editors@unimelb.edu.au Subscribers will receive email abstracts of accepted papers to an address of their choice. For institutional subscription, please email the managing editor at ajl-editors@unimelb.edu.au

Complete published papers may be downloaded at the journal's website at http: //www.philosophy.unimelb.edu.au/ajl/ The journal currently publishes in pdf format.

Submission The journal accepts submissions of papers electronically. To submit an article for publication, send the $\mathrm{LT}_{\mathrm{E}} \mathrm{X}$ source of a submission to a member of the editorial board. For a current list of the editorial board, consult the website.

The copyright of each article remains with the author or authors of that article. 